\title{
La transaction foncière comme mode de régulation en matière de protection contre les inondations. Analyse à partir de deux zones d'expansions de crue : l'île Saint Aubin (Angers) et le déversoir de la Bouillie (Blois).
}

\section{Land transaction, as a mode of regulation for flood management. An insight from two flood retention areas: l'île Saint Aubin (Saint Aubin} Island, Angers) and la Bouillie discharge channel (Blois).

\author{
Mathieu Bonnefond ${ }^{1}$, Marie Fournier ${ }^{2}$, Sylvie Servain ${ }^{3}$, Mathilde Gralepois ${ }^{4}$ \\ ${ }^{1}$ LTSER Zone Atelier Loire - CNRS, E.A. 4630 Laboratoire Géomatique et Foncier, Conservatoire National des Arts et \\ Métiers, mathieu.bonnefond@lecnam.net \\ ${ }^{2}$ E.A. 4630 Laboratoire Géomatique et Foncier, Conservatoire National des Arts et Métiers, marie.fournier@lecnam.net \\ ${ }^{3}$ LTSER Zone Atelier Loire - CNRS, UMR 7324 CITERES, INSA Centre Val de Loire, sylvie.servain@insa-cvl.fr \\ ${ }^{4}$ UMR 7324 CITERES, Université François Rabelais, Polytech Tours, mathilde.gralepois@univ-tours.fr
}

\begin{abstract}
RÉSUMÉ. Construire la résilience de territoires peut passer, entre autres, par la mise en œuvre de stratégies foncières élaborées par les acteurs publics. Dans le cadre de territoires urbanisés marqués par des opérations de densification et de renouvellement urbain, de nombreux cas d'étude permettent d'observer les conditions de mise en œuvre de stratégies de prévention des risques d'inondation qui choisissent pour partie de délocaliser les solutions techniques de gestion des crues, afin de garantir ou d'aménager des zones d'expansion des eaux situées en amont des zones inondables urbanisées dans des secteurs à la vulnérabilité faible ou réduite par un aménagement particulier. La mise en œuvre d'instruments d'action foncière (acquisition, convention, expropriation, etc.) permet la (re)localisation de solutions de gestion de l'aléa hors de la zone inondable concernée. Nos cas d'étude porteront sur la mise en place ou le maintien de Zones d'Expansion de Crue (ZEC) à proximité des villes de Blois (déversoir de la Bouillie) et d'Angers (lle Saint Aubin) en France. A partir de ces deux exemples, nous montrerons que ces actions foncières, entendues comme des " transactions », correspondent à des échanges négociés, qui entrainent une reconfiguration des usages locaux et recouvrent une dimension stratégique qui dépasse la seule question des droits sur le sol. Nous questionnerons les modalités de mise en œuvre de cette transaction foncière et sa participation à la construction d'une résilience territoriale. ABSTRACT. For local governments, the implementation of land tenure strategies may constitute a key component for the reinforcement of territorial resilience. As such, it is now quite common in France to implement flood protection projects based on the preservation of water retention areas, which are located upstream on-going urban development schemes. Such projects are implemented in order to protect the various issues at stake in those densely populated areas. In this context, the use of land tenure instruments (acquisition, local agreements, expropriation, etc...) may facilitate the implementation of those projects. In this paper, we will focus on 2 case studies with the example of flood expansion areas located nearby the cities of Blois (Bouillie spillway) and Angers (Ile Saint Aubin). In both projects, those water retention areas have been restored and/or maintained in order to protect neighboring urban estates, via the implementation of specific land tenure strategies. In this paper, we will describe and analyze those strategies. We will describe how they can be seen as "transactions" (or negotiated exchanges) between local stakeholders, that they may lead to the reorganization of local land uses and often have a strategic dimension which overtakes the mere land rights issue. At last, we will question those transactions on land, as they may constitute a crucial condition for the reinforcement of local resilience.

MOTS-CLÉS. Foncier, Inondation, Loire, Politiques publiques, Régulation, Résilience, Transaction.

KEYWORDS. Land tenure, flood, Loire River, Public policies, Regulation, Resilience, Transaction.
\end{abstract}

\section{Introduction}

Alors que les ouvrages verticaux de contrôle des crues (digues, barrages) se sont multipliés tout au long du $\mathrm{XX}^{\mathrm{ème}}$ siècle sur un grand nombre de cours d'eau en France, on observe depuis le début des années 2000 l'émergence de projets privilégiant une gestion "horizontale» de l'aléa (canaux de décharge, zones d'expansion des crues, bassins de rétention) laissant, lors des crues, les eaux s'étendre de façon contrôlée dans certaines zones inondables, souvent au profit d'espaces urbanisés en amont ou aval [FOU 16a]. Des systèmes de 
contrôle de l'aléa sont ainsi aménagés, dans une très forte proximité avec les espaces urbanisés à protéger ou de manière plus éloignée.

L'article présenté ici s'appuie sur deux exemples récents de restauration ou de maintien de zones d'expansion de crue en amont d'agglomérations urbaines (Figure 1). Il s'agit pour le premier d'un aménagement situé à proximité de la ville de Blois, sur la Loire : le déversoir et le canal de décharge de la Bouillie. Le second correspond aux prairies inondables de l'Île Saint Aubin maintenues pour leur rôle de bassin de rétention des eaux, en amont d'Angers, au cœur des basses vallées angevines (Maine). Ces deux terrains ont été investis à l'occasion d'un projet de recherche européen (JPI Climate TRANS-ADAPT ${ }^{1}$ ), finalisé en octobre 2017. Le projet questionnait les modalités d'implication des acteurs locaux, qu'ils soient institutionnels ou issus de la société civile, dans les projets d'aménagement multifonctionnels dans des espaces dédiés à la gestion du risque d'inondation.

Face au risque d'inondation, les zones étudiées prennent la forme «d'espaces solutions » [BER 02] pour ces deux agglomérations de taille moyenne. Les démarches engagées dans le cadre de ces projets ont permis la (re)localisation de solutions de prévention du risque, en amont des zones inondables urbanisées. Si, dans les deux cas de figure, la localisation des aménagements est envisagée majoritairement dans des espaces à dominante naturelle ou agricole, ces derniers se situent néanmoins à très forte proximité des zones urbanisées où les activités tendent à se diversifier, avec un front d'urbanisation de plus en plus proche.

L'article porte plus précisément sur les instruments d'action foncière (acquisition, convention, expropriation, etc.) qui ont été mobilisés localement afin de mettre en œuvre ces opérations de restauration ou de maintien de zones d'expansion des crues. Elle nous amène à poser la question de l'échelle et de la territorialisation de la résilience, tant du point de vue de la capacité de résistance, d'absorption, de récupération des systèmes urbains face au risque inondation [SER 11] que de celui de l'organisation territoriale [BAR 12]. Nous montrerons en quoi la mobilisation des instruments d'action foncière par les différents acteurs pose les bases d'une résilience technique mais aussi sociale dans ces secteurs (dés)aménagés en zone d'expansion de crue. Derrière la mobilisation de ces outils d'action foncière, se dessinent aussi des enjeux recouvrant une dimension sociale forte. Le parti est alors d'appréhender les actions foncières prises dans leur ensemble comme l'expression d'une transaction sociale [REM 1991; REM 05]. Le terme de "transaction foncière " permet de mettre en exergue l'ensemble des échanges négociés autour de la maîtrise du foncier (évolution des droits de propriété, mise en place de dispositifs de contractualisation, etc...). Les jeux de négociation qui ont lieu dans le cadre de cette transaction n'ont pas uniquement pour consistance un échange autour des droits de propriété, mais expriment également des jeux de pouvoir et de contre-pouvoir autour d'un ou plusieurs problèmes à résoudre (voire des jeux de valeurs). Ces derniers recouvrent ainsi une dimension sociale qui trouve une expression au travers d'actes formalisés en matière foncière. Ces échanges négociés sont des modes de régulation institutionnalisés et territorialisés, en les appréhendant dans leur ensemble, sur un espace déterminé et dans leurs multiples dimensions économiques, sociales et politiques. Si la transaction foncière trouve sa formalisation en matière foncière (vente, bail, etc.), elle révèle un processus de négociation entre acteurs autour d'enjeux ou de problèmes inscrits dans le temps et dans l'espace. Ce sont ces derniers auxquels nous nous attacherons pour cet article.

Trois temps nous permettent de décrypter ces processus. Tout d'abord nous présenterons les projets d'aménagement de Zones d'Expansion des Crues pris comme études de cas pour cet article et observés au regard des enjeux fonciers et d'usages qu'elles supportent. Puis, il s'agira de mettre en lumière les instruments de maîtrise foncière mobilisés dans le cadre de ces projets. Enfin, nous rendrons compte de la dimension territoriale que recouvrent les transactions foncières à l'œuvre localement.

\section{Quand la protection d'un territoire urbain trouve sa solution dans les espaces ruraux et péri-urbains situés à l'amont}

Dans le cadre de territoires urbanisés marqués par des opérations de densification et de renouvellement urbain, de nombreurses stratégies de gestion des risques d'inondation cherchent à délocaliser ou relocaliser les solutions techniques de contrôle des crues en amont, en passant notamment par la mise en place d'actions foncières. Ces stratégies consistent à garantir ou à aménager des zones d'expansion des eaux situées en amont 
des zones inondables urbanisées, dans des secteurs à la vulnérabilité faible (l'Île Saint Aubin à Angers) et, où sont parfois déjà présents des systèmes de contrôle de l'aléa (le déversoir de la Bouillie à Blois).

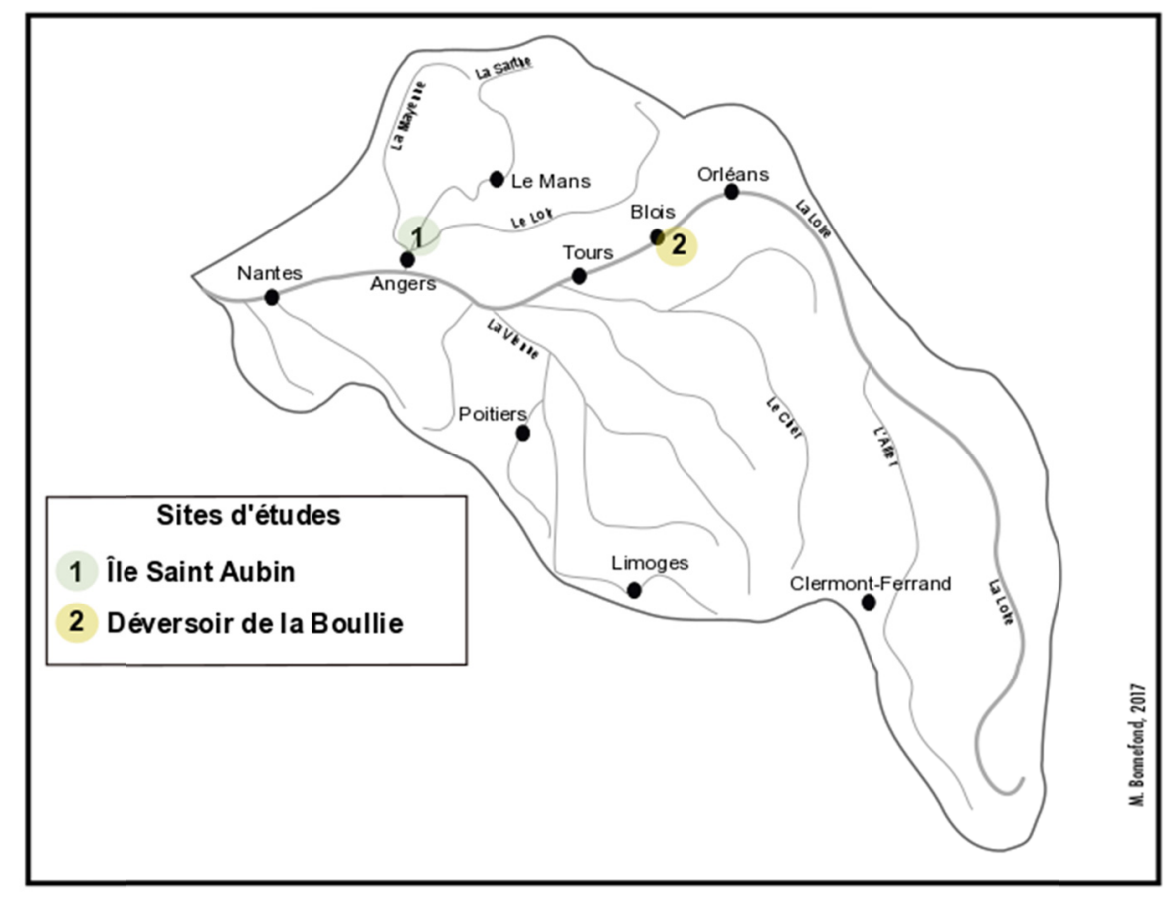

Figure 1. Localisation des sites d'études

Ces stratégies ne s'appliquent néanmoins pas à des espaces exempts de toute activité ou usage et augurent des modifications profondes pour les espaces visés, souvent avec une dégradation des conditions locales et une augmentation de l'inondabilité du périmètre visé.

Les données analysées sont issues d'un travail bibliographique (littérature académique et littérature grise), d'enquêtes de terrain et d'entretiens semi-directifs auprès de l'ensemble des acteurs concernés par ces projets, tant institutionnels qu'usagers ou riverains (une quinzaine d'entretiens par site). Dans cette première partie, nous rendrons compte de la variété des usages qui préexistaient dans les périmètres étudiés avant la mise en œuvre des projets de restauration ou de maintien des zones d'expansion des crues. Plusieurs instruments d'action foncière ont été mobilisés dans les deux cas de figure par les gestionnaires du risque, principalement les communes et leurs groupements, mais aussi par d'autres acteurs institutionnels.

\subsection{Le bœuf et l'oiseau au service de la protection urbaine : le maintien d'espaces ouverts dans l'île St Aubin}

L'Île Saint Aubin, qui s'étend sur près de 600 ha, est située dans les Basses Vallées Angevines, plaines alluviales inondables de 10000 ha, proches d'Angers, au confluent de deux rivières, la Mayenne et la Sarthe (Figure 2). L'ensemble des Basses Vallées Angevines est reconnu et protégé par plusieurs législations environnementales, cette zone étant cruciale en termes de biodiversité pour une grande variété d'espèces, dont le râle des genêts, de prairies et de zones humides (site Natura 2000, Zone Spéciale de Conservation (ZSC) et Zone de Protection Spéciale (ZPS), zone humide RAMSAR). 


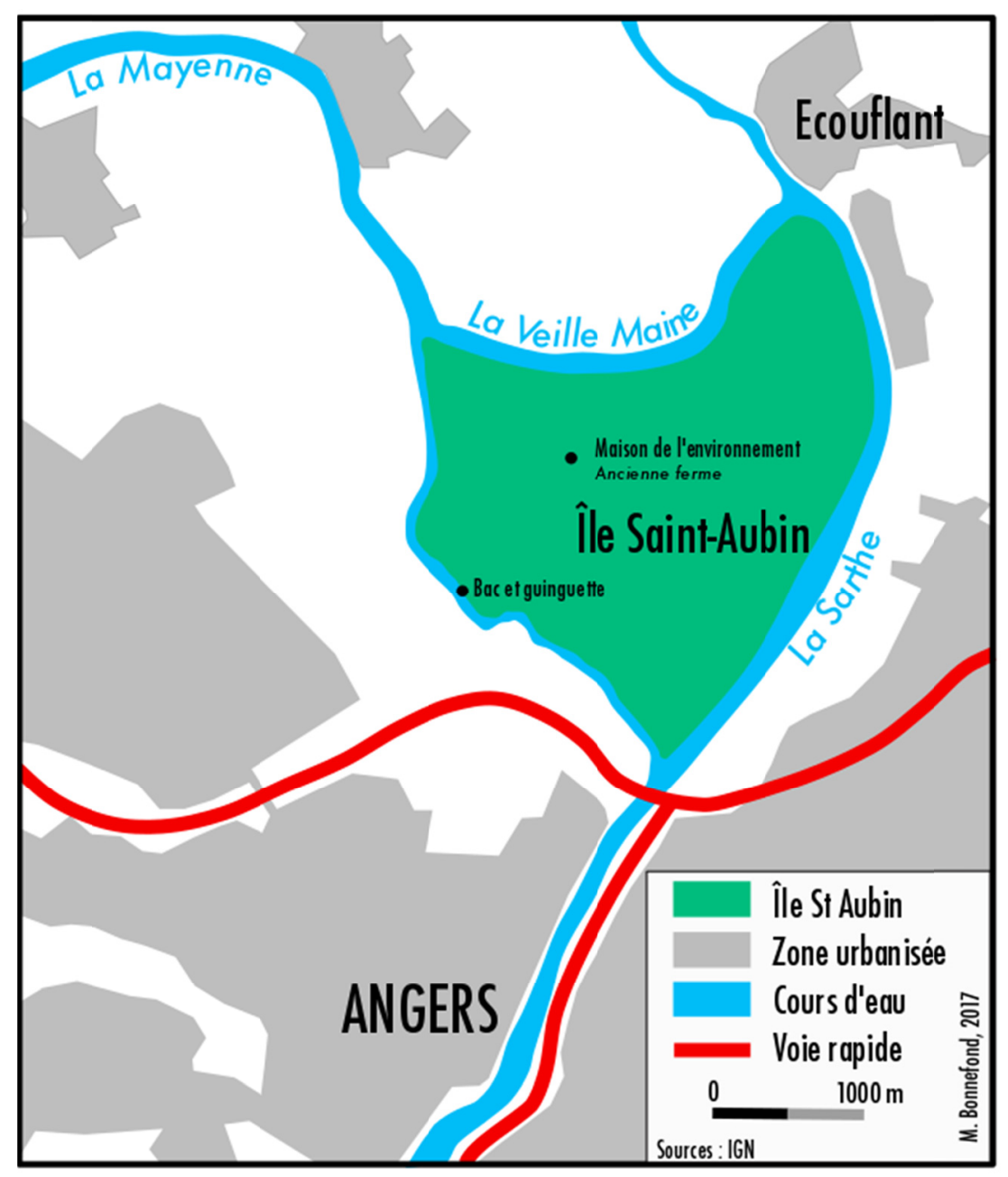

Figure 2. L'Île Saint Aubin

L'Île Saint Aubin se trouve en amont de la ville d'Angers. Elle joue un rôlle d' «éponge » et de zone d'expansion naturelle, réduisant les débits de la Maine avant son passage dans la ville et de fait atténuant, dans une certaine mesure, le risque d'inondation.

Aménagée et drainée au Moyen Âge par une communauté monacale, l'île a conservé jusqu'à nos jours une vocation agricole forte. Valorisés par les moines jusqu'à la Révolution, ces espaces de pâture et de fauche ont ensuite été divisés en parcelles et vendus à des dizaines de particuliers. Jusque dans les années 1970, le pâturage et la fauche y sont restés très actifs. L'activité agricole a néanmoins commencé à décliner, avec un abandon des terres, sur ce parcellaire morcelé. Progressivement, à l'instar de l'ensemble des Basses Vallées Angevines, les propriétaires fonciers se sont détournés de l'île et de son exploitation. A la fin des années 1970, certains propriétaires ont commencé à planter des peupliers, en particulier dans sa partie nord, menaçant l'équilibre de l'île. Ce phénomène a notamment amené des acteurs institutionnels locaux à investir, et surtout à acquérir du parcellaire, avec différents objectifs (au départ relativement éloignés de la problématique du risque). Ainsi, une association locale de chasseurs, liée à la Fédération Départementale des Chasseurs et à l'association nationale Faune Sauvage, a acquis progressivement plusieurs dizaines d'hectares avec pour objectif la protection et le maintien des prairies humides inondables essentielles aux oiseaux migrateurs. Par l'acquisition de parcelles dispersées, l'ambition de l'association est de préserver ce milieu prairial menacé par les plantations de peupleraies [VIL 02 in CHE 15]. Ces dernières engendreraient en effet la fermeture du milieu et compromettraient la migration d'espèces avicoles. Parallèlement, la ville puis l'agglomération d'Angers se sont intéressées à l'Île Saint Aubin comme espace de loisirs. En 1976, la ville d'Angers établit un périmètre de Zone d'Aménagement Différé, qui lui permet d'acquérir plusieurs dizaines d'hectares avec pour objectif une ouverture au public (création d'un bac afin d'accéder au site en traversant la Mayenne, Cf. Figure 3), le développement de l'éducation à l'environnement (Maison de l'île Saint Aubin), le maintien de l'agriculture mais également la protection de la nature et de la biodiversité. 


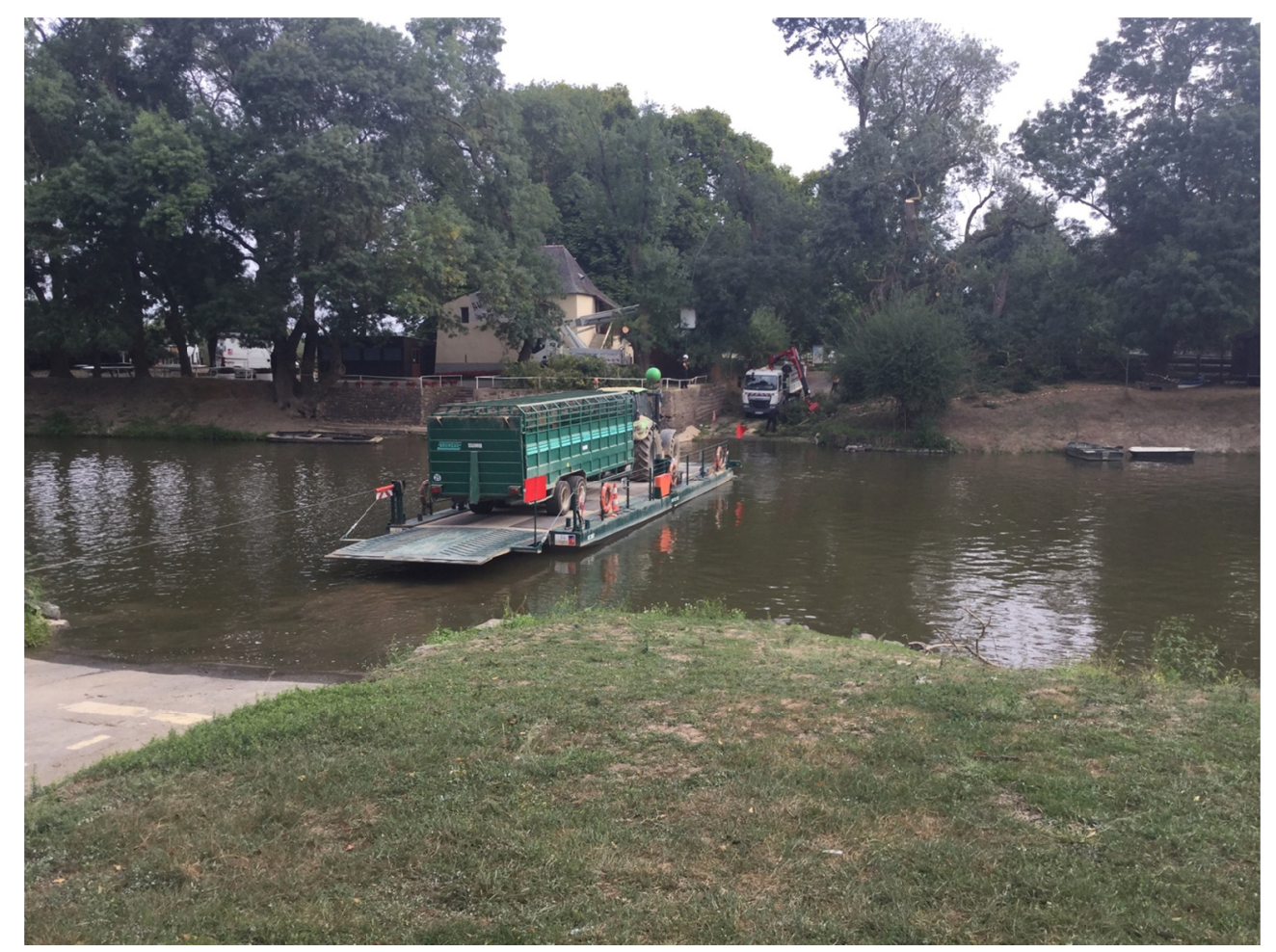

Figure 3. Bac pour accéder à l'île Saint Aubin (Bonnefond, 2016)

Aujourd'hui, l'île est composée de prés clos dans sa partie nord la plus élevée et de prés communs dans sa partie sud inondable. Les activités sont régies par une Association Syndicale Autorisée qui regroupe les propriétaires de l'île et a établi un règlement intérieur de l'île. Ainsi, si les prés communs sont fauchés par leur propriétaire ou leur fermier, le pacage par les animaux est réglementé. Les exploitants doivent s'acquitter d'un ticket pour le pacage de leurs animaux sur les prés communs (Figure 4). Le règlement de l'île a été actualisé en 2010, notamment pour intégrer les activités de loisirs.

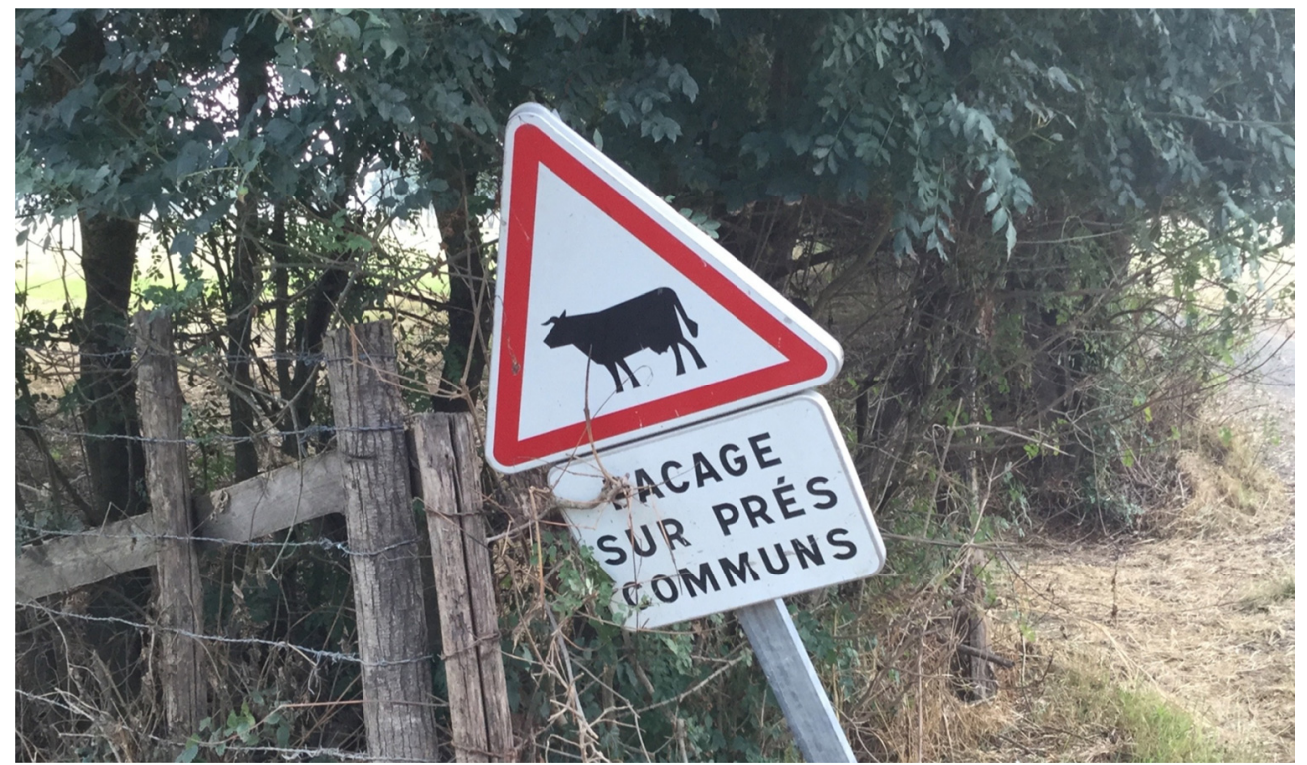

Figure 4. Panneau indiquant le pacage sur prés communs caractérisés par l'absence de clôture (Bonnefond, 2016)

Ainsi, en plus des contraintes liées à l'inondabilité de la plaine protégeant la ville d'Angers, c'est aussi grâce à la présence d'espèces d'oiseaux migrateurs et de prés communs pour le pâturage que l'Île Saint Aubin est aujourd'hui conservée et maintenue en espace non seulement ouvert mais aussi utilisé. L'enjeu majeur pour l'île Saint Aubin mais aussi pour la zone d'expansion concernée par le déversoir de la Bouillie, est de maintenir des espaces ouverts, sans contraintes hydrauliques. 


\subsection{Redonner de la place à l'eau : la restauration d'un ouvrage hydraulique oublié sur le site dit de la Bouillie}

A partir de la fin du XIX $^{\text {ème }}$ siècle et surtout durant la première moitié du $\mathrm{XX}^{\text {ème }}$ siècle, la ville de Blois, comme la plupart des villes ligériennes, a commencé à s'étendre dans la plaine d'inondation de la Loire [SER 14]. La situation de cette ville est néanmoins particulière puisque certains de ces nouveaux quartiers ont investi et progressivement occupé une partie d'un aménagement ancien, prévu pour le contrôle des crues et construit au $\mathrm{XVII}^{\mathrm{eme}}$ siècle : le déversoir et le canal de décharge dit de la Bouillie. Le but de cet ouvrage est d'offrir une section d'écoulement supplémentaire à la Loire en cas de fortes crues et ainsi limiter le débit du fleuve à 3900 $\mathrm{m}^{3} / \mathrm{s}$ dans le lit principal de la Loire lorsqu'elle longe le centre-ville. L'ouvrage compense l'effet du rétrécissement du lit du fleuve au passage du pont Jacques Gabriel. En cas de fortes crues, une partie de l'eau s'écoule ainsi vers l'aval de la vallée, au sud, et rejoint un affluent de la Loire, le Cosson. L'ouvrage est établi sur la digue et construit avec des matériaux robustes à un niveau plus bas que la levée située en amont et en aval; il avait été pensé pour fonctionner pour une crue supérieure à la crue cinquantennale de $3800 \mathrm{~m}^{3} / \mathrm{s}^{2}$ [ROD 08].

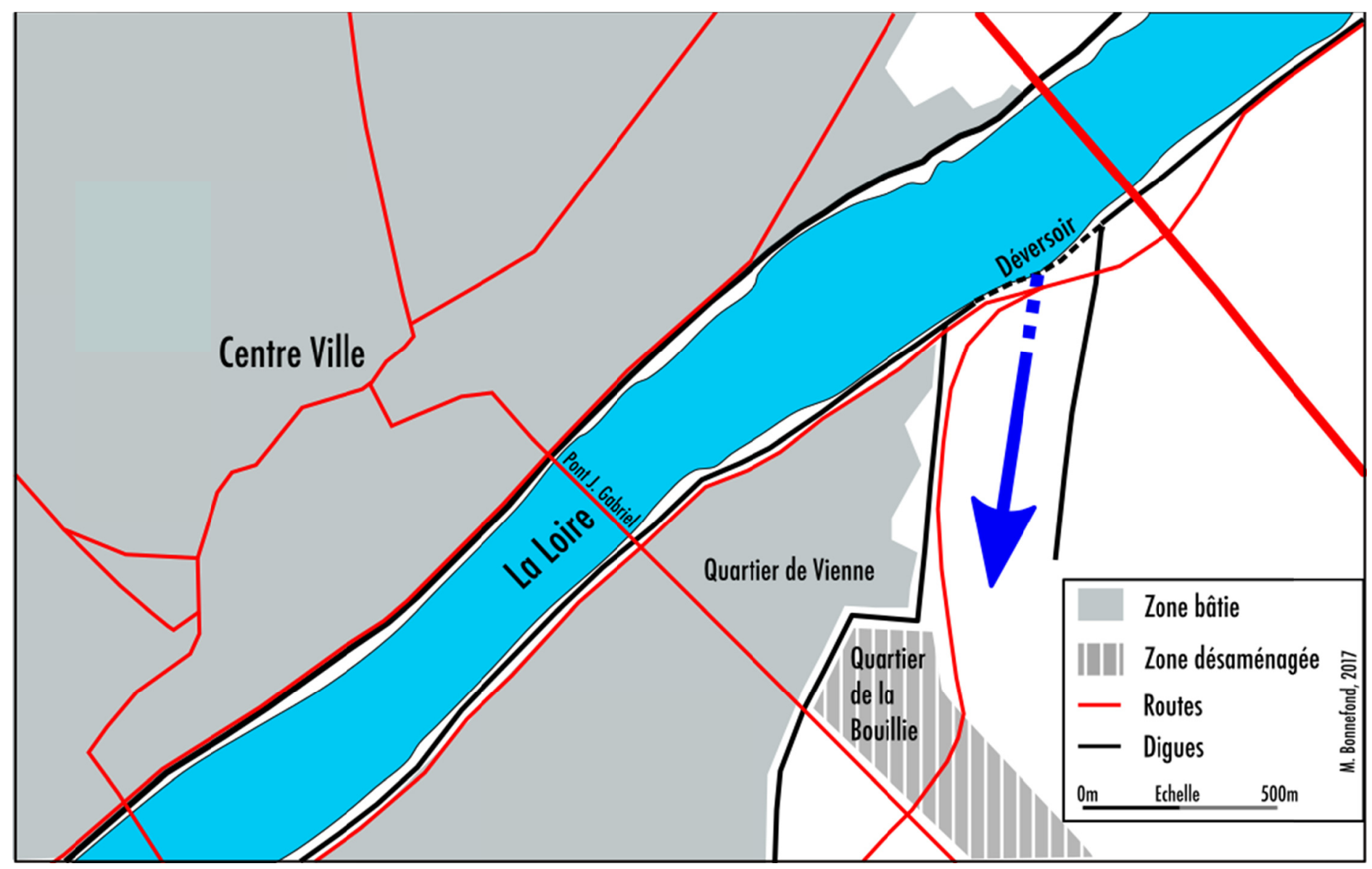

Figure 5. Le déversoir et canal de décharge de la Bouillie

Néanmoins, comme évoqué précédemment, se sont progressivement concentrées au fil du temps un certain nombre d'activités au sein du canal de décharge. Au début des années 2000, le périmètre, dans sa partie amont, était occupé par 135 maisons individuelles et 14 emplacements d'activités employant plusieurs centaines de personnes, mais aussi des terrains de sport et des jardins familiaux. Un camp de gens du voyage est également installé à proximité du déversoir. La ville n'ayant connu aucune inondation majeure au $\mathrm{XX}^{\mathrm{eme}}$ siècle ${ }^{3,4}$, cette urbanisation (au départ informelle) du fond de vallée a pu progressivement être encouragée par un sentiment

\footnotetext{
${ }^{2}$ Station hydrométrique de Blois K4470010 - DREAL Centre (source http://www.hydro.eaufrance.fr/)

${ }^{3}$ Les dernières inondations majeures se sont produites au milieu du XIX ${ }^{\text {ème }}$ siècle en 1846, 1856 et 1866 . La dernière inondation par débordement du déversoir a eu lieu en 1907. La crue centennale de 1856 a atteint 6.78 mètres à Blois. L'eau n'a pas dépassé la côte des 5 mètres depuis les années 1920.

${ }^{4}$ Dans le val de Blois, la vallée de la Loire est protégée des inondations par un système de digues dont l'objectif s'inscrivait dans une perspective de protection du fond de vallée pour y développer des activités agricoles, économiques et urbaines et des lignes de communication.
} 
largement répandu de protection derrière les digues [SER 14]. Au-delà de ce vaste quartier d'habitations et d'activités, le canal de décharge de la Bouillie (Figure 6) constitue avant tout une vaste zone de prairies et de zones humides relativement peu valorisée dans sa partie amont et cultivée dans sa partie en aval.

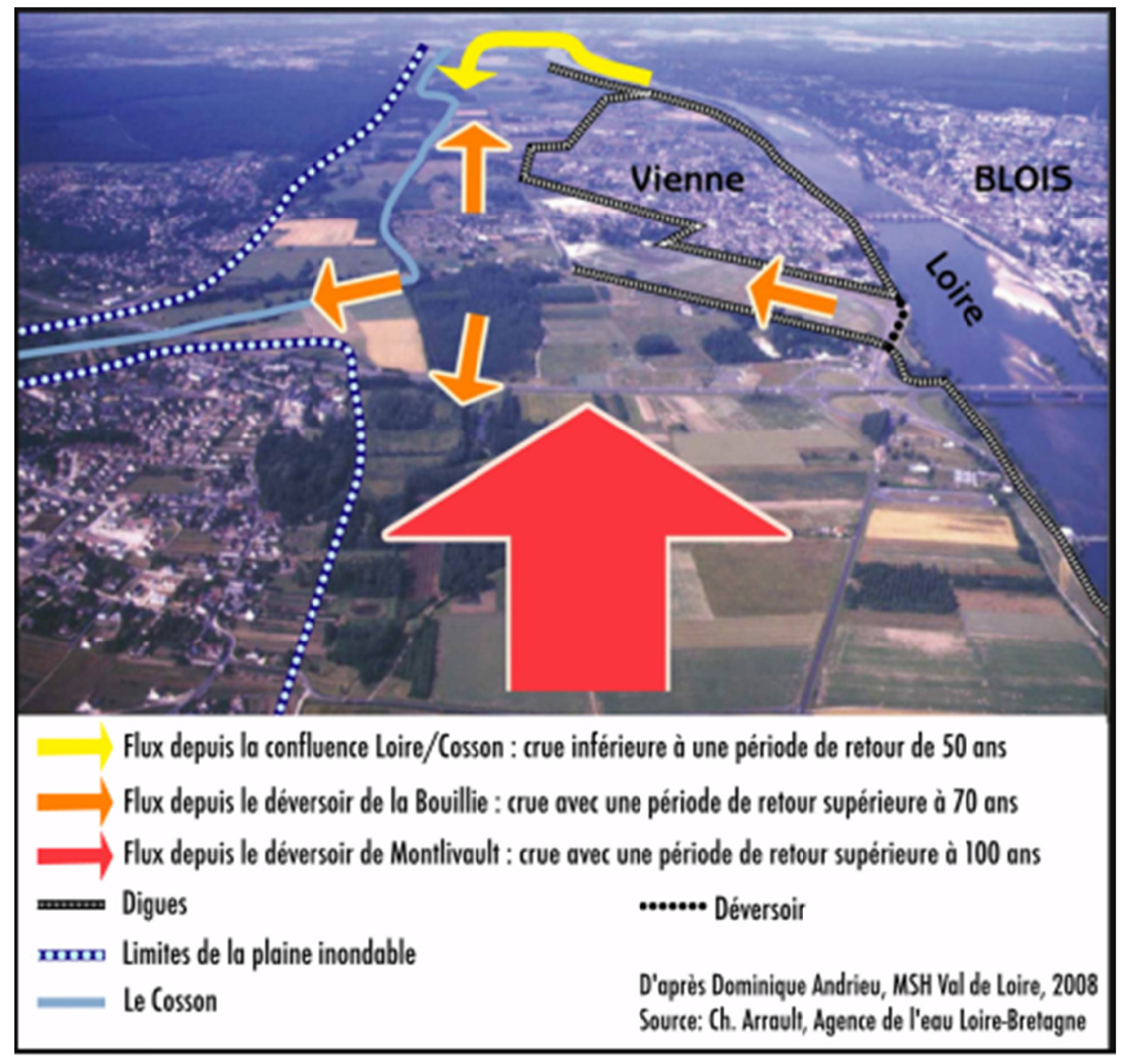

Figure 6. Fonctionnement du déversoir de la Bouille

Le cas d'étude de la Bouillie montre à quel point les aménagements hydrauliques peuvent être oubliés dans le développement des territoires si leur fonctionnement n'est pas entretenu et que leur rôle n'est pas expliqué tant aux collectivités locales qu'aux habitants. Ce cas illustre aussi le décalage qui peut être ressenti entre la localisation d'un ouvrage et le territoire à protéger.

En effet, aux termes de cette première partie, on comprend les potentielles contradictions qui peuvent se trouver lorsque la protection d'un territoire urbain trouve sa solution dans les espaces ruraux et péri-urbains situés à l'amont. Le cas est d'autant plus criant sur l'île Saint Aubin car la grande zone humide est éloignée du centre urbain de l'agglomération d'Angers. Ceci dit, dans le cas de la Bouillie, la proximité entre le déversoir et la rive urbanisée de Blois ne rend pas plus facile la cohabitation car la tension sur le développement est très fort. Dans les deux cas, c'est bien au travers de l'étude de la maîtrise du foncier que s'observent les enjeux du maintien et/ou de la restauration des zones d'expansion des crues.

\section{La maîtrise du foncier, un préalable à la mise en œuvre d'actions de maintien ou de restauration des zones d'expansion des crues}

Pour les deux terrains analysés, la problématique du risque d'inondation et la nécessité d'agir par la maîtrise foncière afin de maintenir ou retrouver des zones naturelles d'expansion des crues se sont affirmées au début des années 2000. Si la maîtrise du foncier par l'acquisition est apparue comme un point de passage obligé, les stratégies d'action ont été relativement différentes. 
2.1. Ile Saint Aubin : des stratégies d'acquisition foncière au fil de l'eau et dans une logique de gré à gré

Dans le cas de l'Île Saint Aubin [FOU 16b], si la nature très fortement inondable de l'île n'a jamais été discutée (l'île étant sous les eaux plusieurs semaines tous les hivers), son rôle dans l'atténuation du risque d'inondation en cœur d'agglomération angevine resurgit à l'occasion de l'inondation majeure qui touche le bassin de la Maine en 1995. Un plan de Prévention du Risque d'inondation (PPRi) a ainsi été mis en place sur la zone de confluence de la Maine en 2009, se substituant au Plan de Surfaces Submersibles de 1958. Dans ce cadre, la préservation des prairies devient un objectif important et les nouveaux boisements sont fortement contrôlés. Le PPRi reconnaît clairement le rôle de l'Île Saint Aubin comme écrêteur de crues en amont de la ville d'Angers. Il empêche toute urbanisation et règlemente le boisement de l'île (règles de distance entre les arbres, règles de hauteur) ${ }^{5}$. Tous les acteurs locaux reconnaissent le lien entre le maintien d'un paysage ouvert par l'agriculture et le rôle essentiel de stockage de l'eau de la zone en période de crue. La partie aval des Basses Vallées Angevines, dont fait partie l'Île Saint Aubin, fonctionne «comme une bassine qui peut stocker des volumes très importants $»^{6}$. Les différentes réglementations établies sur le parcellaire de l'île appréhendent tant les enjeux de biodiversité que de gestion du risque d'inondation. Ainsi, collectivités et associations de chasseurs, désormais propriétaires d'un parcellaire de plus en plus conséquent, contractent, entre autres, des baux ruraux à clauses environnementales avec les agriculteurs de la région pour la fauche de leurs parcelles, intégrant depuis les années 90 des préconisations de fauches tardives notamment en lien avec les mesures agroenvironnementales. Par ailleurs, l'association des propriétaires fonciers, héritage des prés communaux, réglemente le pâturage, mais interdit les plantations d'arbre ou l'aménagement de fossés clôturant les parcelles sur la plus grande partie de l'île. Sur les prairies fermées, les clôtures doivent rester perméables, afin de limiter les obstacles à l'écoulement. Une interdiction de boisement a aussi été prise au niveau départemental par les services de l'État au travers d'un «arrêté de boisement» en 1998, puis en 2005, qui interdit toute nouvelle plantation de peupliers sur l'île Saint Aubin.

Aujourd'hui, par leur stratégie foncière, la ville d'Angers et la communauté d'agglomération Angers Loire Métropole cherchent à articuler la qualité d'éponge naturelle de l'île limitant l'inondation de la partie urbaine de l'agglomération - notamment la ville d'Angers - le développement des activités récréatives et la conservation de la biodiversité. Les acteurs publics (Ville d'Angers et Angers Loire Métropole) et parapublics (Fédérations des chasseurs et Association de pêche) locaux sont propriétaires d'une grande part de l'île (Figure 7).

\footnotetext{
${ }^{5}$ Distance de minimum de 7 mètres entre les arbres et hauteur des arbres limitée à un mètre au-dessus des plus hautes eaux connues.

${ }^{6}$ L'étude des crues dans les Basses Vallées Angevines réalisée en 2012 par le cabinet BCEOM pour le compte de l'Etablissement Public Loire démontre l'importance du stockage dans la partie aval notamment en lien avec les crues de la Loire. 


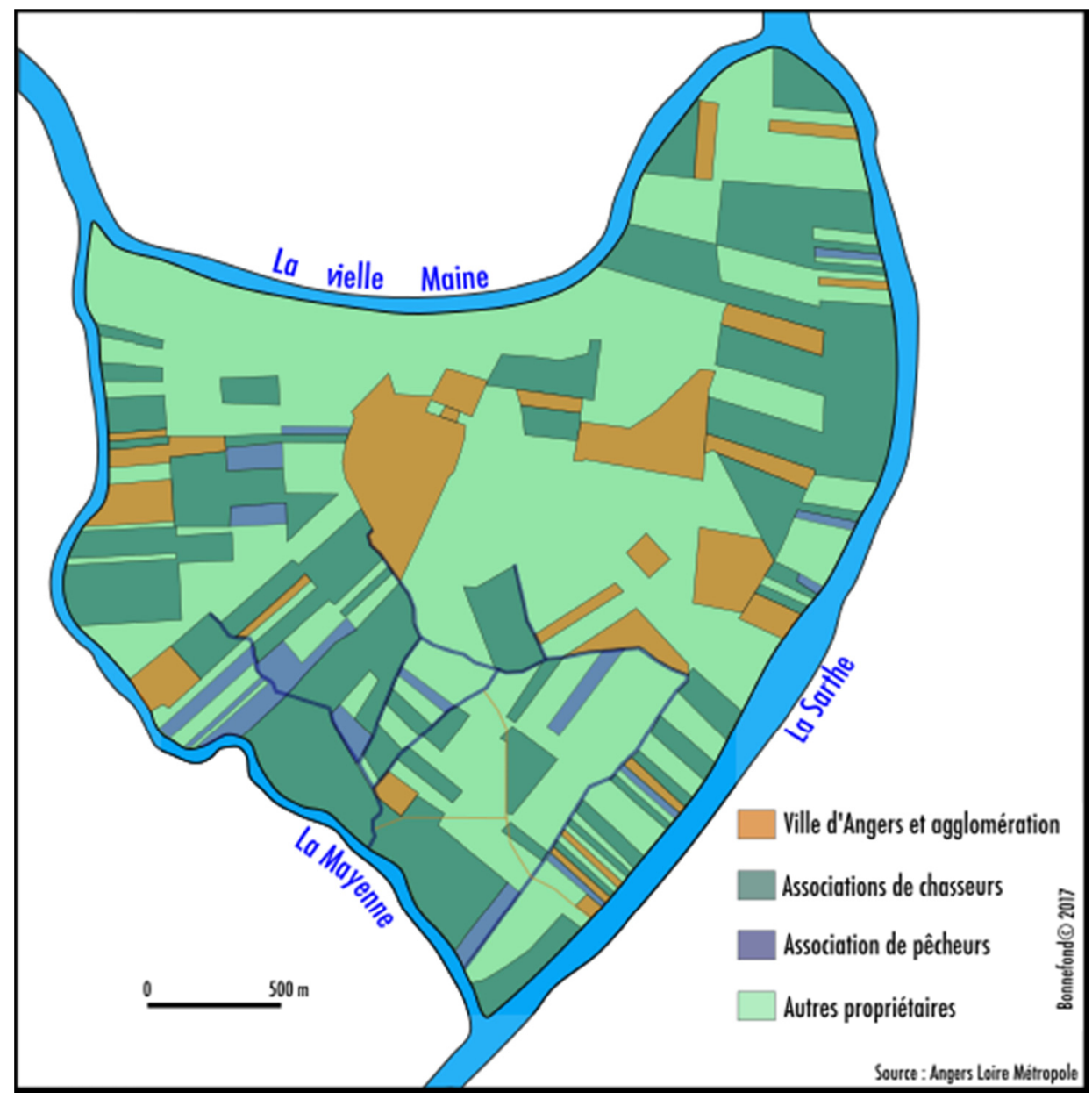

Figure 7. Acquisitions des principaux acteurs institutionnels sur l'île Saint Aubin

Dans le cas d'Angers, les stratégies d'action portées par les différents acteurs institutionnels locaux foncière ne prennent pas de forme conflictuelle. Les achats de parcellaire se font progressivement au gré des opportunités et même si une procédure de ZAD avait été engagée à la fin des années 1970, elle n'a pas conduit à l'achat de l'intégralité de l'île. Le foncier est principalement acquis à l'amiable. Les acteurs instituionnels locaux y ont d'ailleurs des intérêts variés : protection de la biodiversité, tourisme, maîtrise des boisements et bien sûr maîtrise du risque inondation.

\subsection{La Bouillie : une démarche d'acquisition foncière sous tension}

Dans le cas du déversoir et du canal de décharge de la Bouillie, la maîtrise foncière sur la partie amont de l'aménagement devient également un objectif afin de réhabiliter l'ouvrage et son bon fonctionnement hydraulique. C'est par le biais d'une procédure de Zone d'Aménagement Différé (ZAD) que la commune de Blois, puis la communauté d'agglomération Agglopolys, suite à un transfert de responsabilités, a engagé un projet d'acquisition foncière dans le chenal d'écoulement.

Le déversoir et le canal de décharge constituent un enjeu important en matière de protection contre les inondations à l'échelle de l'agglomération blésoise et plus particulièrement pour les quartiers situés en bord de Loire (centre-ville en rive droite et quartier de Vienne en rive gauche). Ainsi, la présence de l'urbanisation dans le canal de décharge de la Bouillie a réduit de $80 \%$ la capacité hydraulique de l'ouvrage. La surélévation de la ligne d'eau de l'ordre de $1 \mathrm{~m}$ qui en résulterait aggraverait le risque de rupture des levées protégeant le quartier voisin, de Vienne [VAL 12]. Ce dernier est un ancien faubourg protégé par un système de digues contre la Loire et son affluent, le Cosson. Il compte 3300 habitants dans 1400 logements et 210 entreprises, mais aussi des équipements publics (écoles, piscine, terrain de sport, station de traitement d'eau, générateur électrique...). Il constitue donc un espace à enjeu important en matière de risque d'inondation. C'est dans ce contexte que la municipalité de Blois, puis Agglopolys, sous la pression forte des services de l'Etat, engage une action d'acquisition foncière du parcellaire sur le périmètre du canal de décharge de la Biouillie [MOR 12]. L'objectif du projet est d'engager, à terme, la déconstruction du quartier (Figure 5), tant pour mettre en sécurité les populations résidentes que pour assurer de nouveau des capacités d'écoulement importantes dans le chenal et ainsi mieux protéger le quartier de Vienne. 
En 2000, le périmètre est arrêté sur la partie amont du canal, englobant ainsi l'ensemble des habitations et des activités économiques présentes sur le site. L'objectif des collectivités locales est d'acquérir le foncier sur un pas de temps de 14 ans, avant de déconstruire l'ensemble du quartier. Les enjeux de sécurité, à l'échelle du quartier, sont réels, mais le projet ne peut aboutir qu'après de longues négociations avec les services de l'Etat en charge de l'élaboration du PPRi, ces derniers exigeant la restauration des capacités d'écoulement dans le canal de décharge avant de permettre la poursuite du développement urbain dans le quartier de Vienne. La ZAD de la Bouillie, très critiquée localement, a fait l'objet d'une opposition riveraine relativement bien organisée, attaquant tant les justifications techniques du projet que les modalités de sa mise en œuvre (Figure 8). Néanmoins, la procédure a abouti au résultat escompté. La majorité des propriétaires a accepté progressivement de voir leurs biens acquis par l'agglomération, celle-ci ayant bénéficié du fonds Barnier pour réaliser les acquisitions.

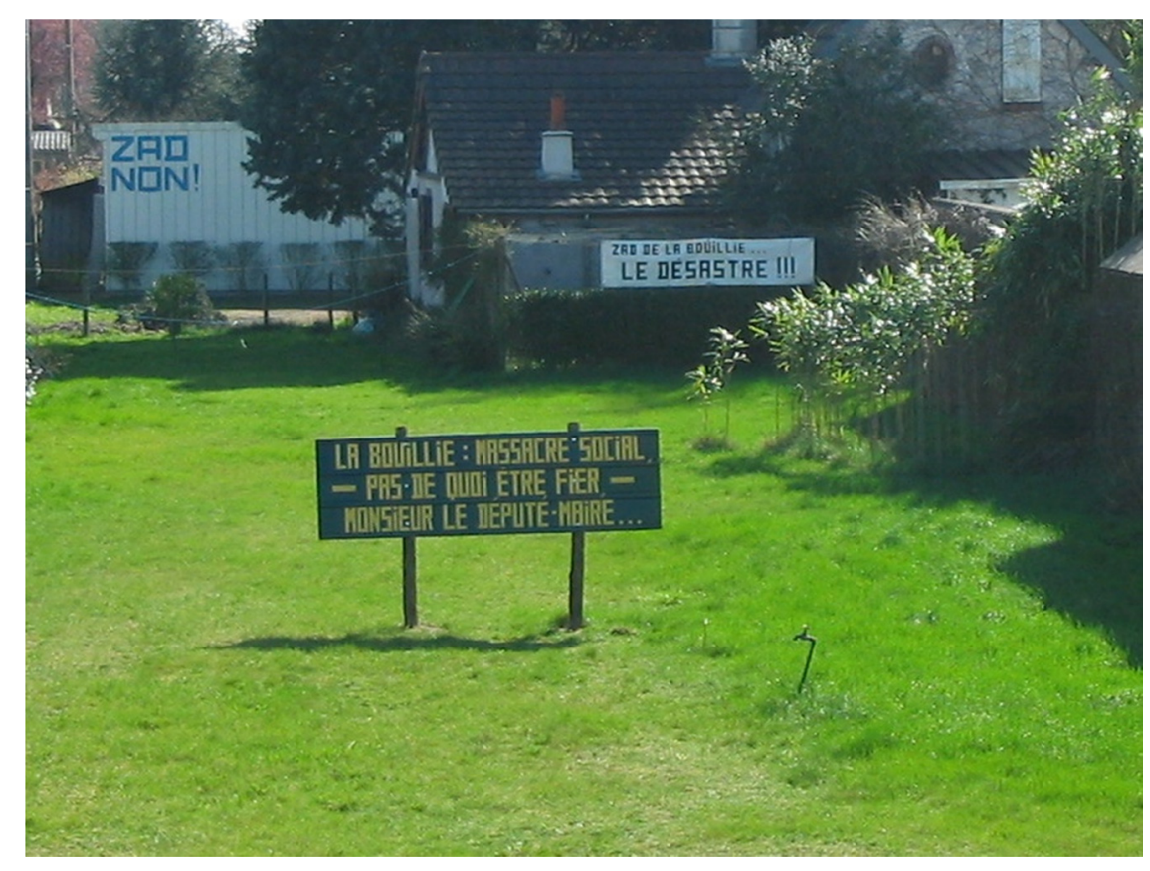

Figure 8. Panneaux témoignant de l'opposition locale à la ZAD (Servain, 2008)

Suite à ces acquisitions, la communauté d'agglomération a rapidement procédé à la destruction des habitations et autres bâtiments qui faisaient obstacle à l'écoulement (Figure 9). Cette opération a permis d'atteindre le premier objectif (hydraulique) du projet. Néanmoins, un certain nombre de questions se posent désormais quant à la valorisation future de ce périmètre. Si de premières propositions ont été formulées [MOR 12], les acteurs institutionnels locaux peinent encore à identifier de nouvelles vocations pour l'ancien périmètre de la ZAD, et au-delà, pour l'ensemble du déversoir soumis au PPRi.

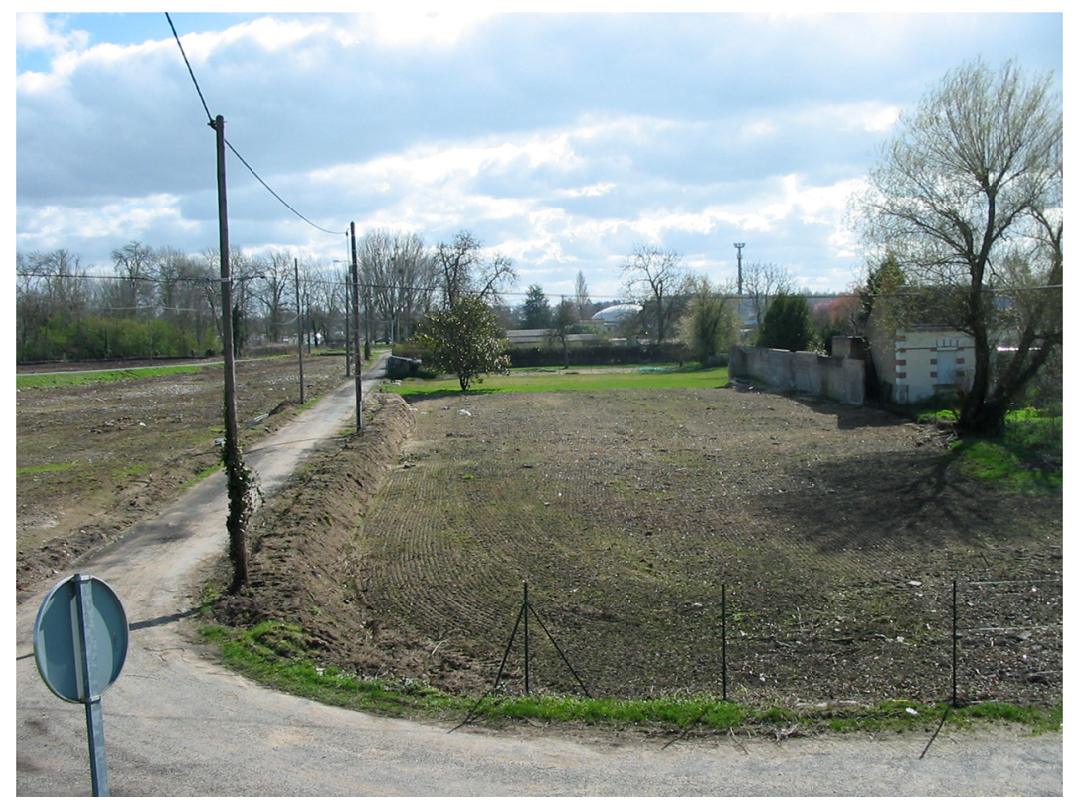


Suite à la création des PPRi et au développement des études relatives à l'évaluation de la vulnérabilité dans les espaces inondables, le développement urbain dans la plaine de la Loire a été fortement remis en question [SER 14]. Depuis le milieu des années 1990, on observe également un changement de paradigme dans l'aménagement du fleuve [HUY 08 ; ROD 10] et une prise de conscience de l'accroissement du risque derrière les digues [FOU 10] [PIG 17]. Dans le cas de la Bouillie, la restauration du canal de décharge s'effectue clairement dans un climat de démarche d'acquisition foncière sous tension. Même si les collectivités locales avaient joué la carte du temps long dans le processus d'acquisition et sous forme de préemption, les incompréhensions ont tout de même été fortes, notamment à cause du manque de décision concrète pour le futur projet d'aménagement en lieu et place de la Bouillie.

\subsection{Au-delà des outils mobilisés, des conditions différentes de mise en œuvre de l'action foncière}

Ces deux exemples montrent bien comment les collectivités locales, lorsqu'elles engagent des opérations de protection contre les inondations, font rapidement face à des problématiques foncières importantes et doivent mobiliser un certain nombre d'outils juridiques impliquant le plus souvent, in fine, l'acquisition du parcellaire. Dans les deux cas de figure, on voit la collectivité passer par l'acquisition pour s'assurer la maîtrise de son projet de gestion du risque. Les deux cas décrits se distinguent cependant à plusieurs niveaux, tout d'abord en termes d'objectifs, tels qu'ils sont portés par les acteurs institutionnels impliqués mais également dans les processus de négociation.

Dans le cas de l'île Saint Aubin, les objectifs sont multiples, de même que les parties prenantes au projet. La problématique de l'inondation s'articule avec d'autres enjeux spécifiques au territoire concerné. Les processus de négociation engagés ne visent pas uniquement à obtenir la maîtrise foncière mais participent d'une dynamique collective locale plus large. Par ailleurs, les démarches d'acquisition foncière sont engagées sur l'Île Saint Aubin depuis désormais près de 40 ans (périmètre de ZAD en 1976). Progressivement, les acteurs institutionnels locaux (Associations de pêcheurs et de chasseurs et collectivités locales) se sont portés maîtres d'une partie du parcellaire par le biais d'acquisitions à l'amiable ou dans le cadre du droit de préemption lié à la ZAD, sans qu'il ait eu obligation de recourir ou simplement menacer d'utiliser la voie de l'expropriation. Ces opérations foncières ont eu lieu dans un contexte local de déprise agricole, où le parcellaire perdait progressivement de son intérêt au regard des évolutions de l'agriculture, mais où une variété d'enjeux et des priorités nouvelles commençaient à s'exprimer (biodiversité, loisirs, paysage, etc...). Lorsque la problématique du risque resurgit au milieu des années 1990, cette dernière s'impose donc sur un périmètre déjà fortement investi par une variété d'acteurs publics en charge de diverses problématiques environnementales. Le passage par la maîtrise foncière relève plus de la mise en œuvre des stratégies variées pour ces acteurs institutionnels que de la mise en œuvre d'une politique locale cohérente en matière de gestion des inondations. La maîtrise foncière permet à ces acteurs de légitimer leur action, tout en l'articulant avec des outils réglementaires dont ils n'ont pas la maîtrise comme le PPRi ou le règlement de boisement, ou des outils incitatifs telles que les mesures agro-environnementales pour le maintien des pratiques agricoles d'élevage extensif. Les processus de négociation sur l'île Saint Aubin s'inscrivent dans une logique de formation de coalitions entre des acteurs aux objectifs différents mais qui cherchent en permanence à s'accorder, de façon à gérer cet espace autour de quelques objectifs communs - maintien de l'espace ouvert et de l'élevage - et de principes communs recherche de consensus, articulation et équilibre entre les usages.

Dans le cas du déversoir de la Bouillie, la nécessité de l'acquisition s'impose d'autant plus que la zone est occupée par des habitations, des entreprises et d'autres équipements. La collectivité locale, sous l'insistance des services de l'Etat, a avant tout un objectif de désaménagement de la zone qu'elle considère d'intérêt général au regard des enjeux humains et matériels présents. La maîtrise du foncier est un outil pour atteindre cet objectif et elle fait le choix d'un outil puissant pour cela. En effet, face à un chenal d'écoulement obstrué par plus d'une centaine d'habitations, la procédure de ZAD engagée en 2000 ambitionne d'aboutir à l'acquisition totale du parcellaire, avec la potentialité d'une expropriation en fin de dispositif. Actuellement, seules quelques rares maisons demeurent, dont les propriétaires ne sont néanmoins plus inquiétés. Le processus est donc beaucoup plus rapide et est à l'œuvre dans une partie de l'agglomération relativement proche (et donc attractive) du centre-ville. En outre, il est dominé par une problématique unique : le contrôle de l'aléa, qui limite l'investissement des autres enjeux propres à ce site.

In fine, si ces actions foncières relèvent du droit des biens, elles mobilisent des objectifs et stratégies de l'action publique et par là interrogent les frontières entre le droit privé et le droit public. La maîtrise foncière devient un réel instrument de politique publique et permet aux acteurs publics de légitimer leur intervention par 
le droit de propriété. Elle permet l'articulation entre l'action publique relevant généralement du droit public et les usages de l'espace relevant plus du droit privé. En cela, elle est au cœur d'un processus de négociation entre acteurs à l'échelle du territoire et d'une transaction territoriale. Si elle s'observe bien sur le site d'Angers, elle n'en demeure pas moins présente sur le site de la Bouillie, en particulier entre l'Etat, les collectivités locales et certains acteurs institutionnels locaux, comme la Chambre d'Agriculture.

\section{Transaction foncière, condition d'une territorialisation de la résilience ?}

Les Zones d'Expansion des Crues (ZEC) sont avant tout des dispositifs qui visent à la réduction ou la nonaggravation du risque inondation. Elles peuvent donc être appréhendées en tant que levier de la résilience urbaine, entendue comme la capacité des villes à absorber une perturbation puis à récupérer ses fonctions à la suite de celle-ci [LHO 10]. Les ZEC que constituent l'Île Saint Aubin ou le déversoir de la Bouillie relèvent bien d'une stratégie technique visant à limiter le degré de perturbation du système par une meilleure capacité d'absorption [SER 11]. Dans cette acception, ces dispositifs, s'ils sont pensés depuis, par et pour la ville, constituent un composant à part entière du système urbain alors qu'ils se localisent à première vue à sa périphérie. En absorbant une partie de la perturbation, ils contribuent à la fois à la résilience de temps court et de temps long [TOU 12]. Les stratégies développées dans les deux cas présentés visent bien à limiter la perturbation par l'expansion de la crue et relèvent de décisions et d'orientations "données à la ville dans l'optique de mieux la préparer à maintenir ses fonctions face à une perturbation » [TOU 12]. Toutes deux s'inscrivent dans la durée et participent ainsi à la mise en place de mécanismes de résilience du système urbain face à des perturbations futures, incertaines, mal évaluées, inconnues.

Cependant, on peut constater que la résilience prend des formes différentes dans ces deux territoires. Si l'on peut parler de résilience technique dans les deux cas, la mise en place de ces dispositifs et l'action foncière qui en découle doivent aussi être analysées dans une approche plus sociale et territorialisée de la résilience [REG 12 ; BAR 12]. De ce point de vue, nos deux exemples révèlent des modes d'intégration territoriale des projets différents d'un territoire à l'autre. Saisir ces processus par le prisme de la transaction foncière et sa territorialisation nous amène donc à investir les projets de ZEC observés sous d'autres angles, à questionner les instruments juridiques de maîtrise du foncier mobilisés et enfin à observer leurs effets territoriaux. En particulier, nous montrons dans cette dernière partie la capacité intégratrice de ces aménagements et les enjeux de la transaction foncière à l'œuvre tant vis-à-vis des usages préexistants qu'en termes de construction d'une gouvernance locale.

\subsection{Transaction foncière et mise en place d'une logique " multi-usages " comme condition d'une résilience territoriale}

La mise en œuvre des actions de maîtrise foncière en zone inondable entraîne une reconfiguration des différents usages (agriculture, pêche, chasse, activités de loisirs, etc.) et recouvre une dimension stratégique qui dépasse la seule problématique du contrôle de l'espace et de ses usages [BON 2009 ; BON 13].

Observées dans leur ensemble, les actions de maîtrise foncière apparaissent comme des actions collectives orientées certes par le droit mais qui mobilisent une diversité d'acteurs publics et privés. Ces actions peuvent également être appréhendées comme une transaction ayant pour objet le foncier et qui relèvent de négociations intégrant et dépassant la somme des actions individuelles. Elles renvoient à des pratiques réglementaires collectives résultant de compromis sociaux et territoriaux complexes, plastiques, renégociés et renégociables. Enfin, elles impliquent des jeux de pouvoirs et de valeurs entre des acteurs et groupes d'acteurs aux objectifs différents. La négociation qui a lieu dans le cadre d'une transaction foncière n'a pas uniquement consistance en matière de maîtrise foncière, elle suppose un jeu de pouvoirs et de contre-pouvoirs autour d'un ou plusieurs problèmes à résoudre, dont ici la protection contre les inondations. Se centrant sur la régulation des conflits, la transaction sociale est un processus qui comprend à la fois de l'échange et de la négociation, du rapport de force et de l'imposition. Elle renvoie à la fois à des conflits d'intérêt, mais aussi à des conflits de valeur et des jeux d'acteurs [GIB 09]. Ainsi, « il y a un double mouvement : une liaison tenant à un enjeu partagé et un espace de séparation, de lutte et d'engagement réciproque » [REM 13]. Des enjeux s'enchevêtrent pour déboucher sur un compromis pratique, formalisé par un échange autour des droits de propriété en termes de droits réels (acquisition) et droits personnels (contrat de location). La transaction foncière est donc à la fois un processus et un produit. Elle relève d'un processus construit par le jeu des acteurs en présence pour faire avancer un problème social qui dépasse le bien immobilier en jeu. Le résultat de ce processus est un produit (transactionnel) qui trouve pour partie une formalisation dans l'échange autour des droits de propriété. Il relève 
donc d'une certaine dynamique, qui est inscrite dans le temps et l'espace. La transaction foncière implique également des interactions entre acteurs en matière sociale, politique, économique, etc... qui sont inscrites dans l'espace et le temps. Cela n'intervient pas seulement autour des enjeux de la gestion et la protection du risque inondation, mais aussi en lien avec d'autres problématiques comme le maintien de l'élevage, la protection de la nature ou la valorisation touristique.

Dans les deux cas de figure qui nous intéressent ici, les communes et EPCI menant les projets font face à des usagers implantés de longue date sur les sites. Les dynamiques locales ne sont néanmoins pas les mêmes. À Blois, de nombreux résidents demeuraient dans le quartier depuis plusieurs décennies et un vrai tissu économique existait sur le site, sans même évoquer les autres usagers du déversoir (gens du voyage, exploitants agricoles, utilisateurs de sites sportifs). Le quartier de la Bouillie, et plus encore le quartier voisin de Vienne, apparaissaient au milieu des années 1990 comme des espaces attractifs, avec des atouts variés et où Agglopolys ambitionnait d'engager un certain nombre de projets [ROD 04]. La redéfinition du risque par les services de l'Etat et l'obligation qu'ils imposent aux collectivités locales de restaurer la ZEC vient geler un territoire situé en zone péri-centrale de Blois, qui faisait l'objet d'ambitions multiples, localement, au niveau urbanistique. À Angers, la dynamique est différente. Un certain nombre d'exploitants viennent valoriser les parcelles de l'île Saint Aubin depuis plusieurs décennies, dans une longue tradition locale, mais l'île est en transition depuis la fin des années 1970. Les difficultés liées aux pratiques d'élevage et plus généralement celles qui peuvent exister pour la valorisation de l'île font qu'elle a connu des phénomènes de déprise durant les dernières décennies. En parallèle, comme évoqué précédemment, d'autres enjeux émergent, en particulier les enjeux environnementaux.

Ces deux démarches prennent donc leur genèse dans des contextes territoriaux différents, qui influent forcément sur la forme que peut prendre la transaction foncière, localement, entre les porteurs des politiques publiques de gestion du risque, les autres acteurs institutionnels et les usagers. À Blois, les opérations de maîtrise foncière engagées ont avant tout comme objectif de faire disparaître un certain nombre d'enjeux (et donc d'usagers) sur un site particulièrement convoité localement. La réception de ces opérations d'acquisition foncière est en grande partie défavorable. À l'inverse, sur l'Île Saint Aubin, les logiques d'acquisition foncière s'inscrivent dans une ambition de maintien et de soutien des usages préexistants.

\subsection{La transaction foncière, moteur d'une redéfinition de la gouvernance locale dans les Zones d'Expansion des Crues}

Du fait de la complexité croissante des jeux d'acteurs au niveau local, avec notamment l'implication de plus en plus forte des différents usagers dans les processus de décision, se pose également la question de la régulation et de la gouvernance des ZEC observées. La gouvernance de ces territoires se trouve enchâssée dans une multitude de niveaux de gouvernance (européen, national, régional, local, etc.) [TOR 11]. Ainsi, interviennent différents acteurs institutionnels, chacun porteur d'objectifs spécifiques. Les acteurs privés investissent également les sphères de décision. Il en résulte des exigences de coordination ainsi que la multiplication des structures de gouvernance territoriale [TOR 11]. C'est dans ce contexte qu'un espace local de décision se dessine, associé à une production de règles négociées formant un ordre juridique localisé [MEL 17 ; BOU 06].

Dans les deux cas qui nous concernent, la gouvernance des ZEC prend des formes distinctes. Tout d'abord, si le portage des projets est le fait des communes et surtout des communautés d'agglomération dans les deux cas (Agglopolys pour le site de la Bouillie, Angers Loire Métropole pour l'Île Saint Aubin), le contrôle des services de l'Etat apparaît beaucoup plus fort sur l'agglomération blésoise. L'ampleur des enjeux humains en présence sur le site, renvoyant à une problématique de sécurité civile forte, la localisation de la ville sur un des grands fleuves français et les responsabilités juridiques à l'œuvre, sont autant d'éléments qui peuvent expliquer une présence des services de l'Etat plus marquée sur le site de la Bouillie. L'Etat reste en effet encore aujourd'hui le principal propriétaire des digues de la Loire et donc responsable de leur bon état. In fine, il apparaît sur le site que l'affirmation d'une fonction unique, dominante, vis-à-vis d'autres usages potentiellement préexistants, limite les possibilités d'ouverture de la gouvernance locale. Ainsi, si des initiatives locales ont été engagées pour proposer des aménagements multi-fonctionnels (paysagers, agricoles, etc...) dépassant la seule gestion de l'aléa, aucune initiative d'ampleur n'a pu encore être engagée ${ }^{7}$ sur le site ayant fait l'objet de la procédure de ZAD. Quelques stigmates du conflit restent d'ailleurs encore apparents, que ce soit

\footnotetext{
${ }^{7}$ Quelques aménagements paysagers marquant la présence de l'eau (pièces d'eau, noue, etc.) vont être réalisés à proximité du déversoir de façon à marquer son rôle dans le paysage.
} 
dans une logique d'opposition (bannières de contestations) ou dans une volonté de conserver des traces de l'histoire du site (préservation de pans de murs ou traces des maisons détruites).

Le jeu d'acteurs apparaît relativement resserré autour des gestionnaires du risque (services de l'Etat, communes et intercommunalité) à Blois et ancré autour de la problématique de la sécurité des ouvrages, tandis qu'il tend à prendre en compte la variété des usagers présents sur l'Île Saint Aubin. Sur cette dernière, la fonction de zone d'écrêtement des crues ne constitue qu'une fonction parmi d'autres et les acteurs institutionnels impliqués tant dans la gestion et la maîtrise du site sont nombreux : fédération de chasse, association de pêcheurs, chambre d'agriculture, etc... Il en découle une gestion de la ZEC ouverte à une variété d'enjeux, au-delà de ceux spécifiquement liés au contrôle de l'aléa naturel. Par ailleurs, la gouvernance de l'île est structurée autour d'une institution ad hoc permettant à la majorité des parties prenantes, tous propriétaires fonciers, d'être représentées. Ainsi, une association syndicale a la charge de la réglementation des activités sur l'île. La qualité de propriétaire est un imposé pour être membre de l'association. Il est intéressant de voir qu'en étant propriétaires d'un parcellaire conséquent, les acteurs institutionnels s'assurent une capacité d'influence sur la structure (le nombre de voix étant défini au prorata du parcellaire détenu). Si les acteurs institutionnels locaux cherchent à la fois à peser dans les décisions au travers des surface qu'ils détiennent (droit de vote), ils ne recherchent pas pour autant à occuper une position hégémonique qui irait à l'encontre de leurs objectifs, qui dépendent du maintien des activités agricoles. Le président de l'association reste encore aujourd'hui un exploitant agricole, également propriétaire d'un parcellaire important. Un jeu complexe est à l'œuvre dans la gouvernance de l'île marqué par une certaine interdépendance entre les différents acteurs, à la fois dans la prise de décision et dans la réalisation de leurs objectifs.

In fine, alors qu'il a pu être montré que dans certains cas la normativité des PPRI peut constituer un frein à la mise en place de la résilience [GRA 12] et que la gestion des risques n'est plus une simple politique de prévention portée par l'Etat mais la résultante d'un processus de décision collective [BAR 13], les stratégies foncières peuvent apparaitre plus intégratrices. La transaction foncière quand elle est opérante peut constituer un vecteur de solidarités territoriales entre les zones qui seraient inondées et celles qui seraient hors d'eau car protégées. Si la transaction foncière pour la mise en place de Zones d'Expansion des Crues s'inscrit dans cette transformation et relève d'une stratégie d'intégration transversale, elle est aussi l'expression des jeux de pouvoirs entre les acteurs et de leur asymétrie. La question de la gestion du risque est ainsi étendue aux problématiques territoriales. Le risque apparaît comme un liant territorial et est alors examiné comme une composante à part entière du système socio-territorial [NOV 08; REG 06]. En appréhendant ensemble la transaction foncière combinée aux différentes qualifications juridiques de ces espaces (ZAD, PLU, PPRi, Natura 2000, etc.) notre travail montre que ces processus négociés produisent une forme de "territoires d'action » [MEL 08], en cela que l'action collective produit du territoire. Cette transaction foncière participe de la construction de territoires d'action et joue un rôle renforcé dans la construction des relations à l'espace des acteurs publics, des habitants et des usagers. Dans une certaine mesure cette transaction foncière contribue à produire des nouveaux «territoires du risque » [NOV 02] qui dépassent les dimensions techniques de la cartographie et juridiques des zones réglementaires. Nous pourrons ainsi parler de transaction territorialisée, correspondant à une transaction sociale dont les processus et les produits sont territorialisés. Un jeu s'établit dans cette transaction, entre la territorialisation des processus et les effets territoriaux de leur produit. La transaction foncière permet une relation réciproque entre la ville soumise au risque et la Zone d'Expansion des Crues. On assiste dans une certaine mesure au travers de l'intégration des enjeux de la ville liés à la question du risque inondation par les acteurs et usagers de la ZEC à l'émergence d'un territoire d'action facteur de résilience territoriale.

L'analyse proposée dans cet article conduit à mettre l'accent sur la transaction foncière, entendue comme une activité normative faite d'interactions multiples entre des acteurs publics et privés qui considèrent davantage le droit comme une ressource stratégique que comme une donnée tangible [COM 09]. Le passage par la transaction foncière apparait ainsi comme un véritable mode de régulation, notamment dans une logique d'intégration de l'action publique environnementale dans les territoires, qui dépasse la simple production de règles secondaires [LAS 07] de mise en œuvre des politiques publiques. Un jeu complexe émerge entre les logiques institutionnelles et les stratégies d'acteurs produisant des modes de gouvernance mobilisant les acteurs publics et privés dans un processus de négociation/renégociation permanent. La transaction foncière produit également des relations de proximité, dont l'objectif dépasse les enjeux sectoriels auxquels elle est liée pour s'insérer dans les processus de gouvernance des territoires [TOR 11 ; LEL 05 ; PAS 07]. La transaction foncière prise dans son ensemble peut être appréhendée comme une transaction sociale [REM 1991; REM 05] car elle dépasse les simples dimensions juridiques (droit des biens) ou économiques (marché foncier: valeur des 
propriétés, valeur d'usage) de la négociation comme le montrent les exemples analysés. La transaction foncière correspond alors à des situations d'interaction particulières et structurées.

\section{Conclusion}

Les deux cas d'étude proposés dans cet article laissent apparaître une inscription territoriale différente de la transaction foncière à l'œuvre. Cette territorialisation est importante, car si elle se formalise par des actes de maîtrise foncière dans la ZEC, elle implique des acteurs, des enjeux et des processus qui dépassent cette zone. A Blois, c'est une stratégie d'exclusion des usagers habitant l'espace sur une partie restreinte à fort enjeu (le quartier de la Bouillie) et l'absence d'intervention foncière dans le reste de l'emprise du déversoir qui prévaut, avec un projet global qui tarde à se concrétiser. Les différents usages et usagers ont certes été identifiés, ainsi que les orientations d'un futur projet en zone inondable - dont le développement des activités agricoles - mais la mise en œuvre se heurte à des difficultés. Dès lors, le cadre de la transaction foncière démontre les limites des processus engagés à Blois. A l'inverse, en ce qui concerne l'île Saint Aubin, nous assistons à une transaction foncière qui cherche à inclure les usages compatibles avec les objectifs de l'action publique. Dans cette recherche d'inclusion, les négociations à l'œuvre dans la transaction débouchent sur des compromis et de potentielles synergies entre les impératifs de gestion du risque d'inondation dans sa dimension technique et les impératifs liés à l'utilisation de l'espace. La ZEC de l'Île Saint Aubin n'est ainsi pas neutralisée dans une perspective monofonctionnelle - la protection contre les inondations - mais est porteuse d'usages multiples dont la compatibilité est assurée au travers de négociations permises par la transaction foncière. L'analyse au prisme de la " transaction » dans sa consistance sociale et territoriale, montre bien en quoi la mise en œuvre de l'action foncière dépasse la simple maîtrise sur le parcellaire et participe à des processus plus larges de négociation. Les deux cas analysés mettent en exergue l'intérêt de penser l'action foncière non pas comme un simple outil à disposition des gestionnaires de ces espaces, mais comme un processus complexe participant de la gouvernance locale.

\section{Bibliographie}

[BAR 13] BARROCA B., DINARDO M. ET MBOUMOUA I., «De la vulnérabilité à la résilience : mutation ou bouleversement? », EchoGéo [En ligne], 24 |, 2013.

[BAR 12] BARROCA B., SERRE D. ET YOUSSEF D., « Le concept de résilience à l'épreuve du génie urbain », VertigO -la revue électronique en sciences de l'environnement [En ligne], Volume 12 Numéro 2 | septembre 2012, 2012 .

[BON 09] BONNEFOND M., Les modes de régulation des usages des espaces naturels en France et au Mexique. Analyse des cas de la Brenne et du bassin du Tepalcatepec. Thèse de doctorat en aménagement de l'espace-urbanisme, Université de Tours, 566p., 2009.

[BON 13] BONNEFOND M. ET FOURNIER M., « Maitrise foncière dans les espaces ruraux. Un défi pour les projets de renaturation des cours d'eau ». Revue d'Economie rurale, numéro 334, 2013/2, pp. 55-68, 2013.

[BOU 06] BOURDIN, A., M.-P. LEFEUVRE ET P. MELE, «L'élaboration des règles du jeu urbain », dans A. Bourdin, M.-P. Lefeuvre et P. Melé (dir.), Les règles du jeu urbain, entre droit et confiance, Paris, Descartes et cie, p. 21-51, 2006.

[BER 02] BERDOULAY V., SOUBEYRAN O., L'écologie urbaine et l'urbanisme : aux fondements des enjeux actuels, La Découverte, 2002.

[CHE 15] CHERRUAULT J., Comment mettre en place une gestion hydraulique intégrée en zone humide? Application à l'île Saint Aubin, Angers. Travail de fin d'étude de l'Ecole Supérieure des Géomètres et Topographes, Conservatoire National des Arts et Métiers, Le Mans, 2015.

[FOU 10] FOURNIER, M., Le riverain introuvable. La gestion du risque d'inondation au défi d'une mise en perspective diachronique. Une analyse menée à partir de l'exemple de la Loire, Thèse de doctorat en aménagement de l'espaceurbanisme, Université de Tours, 431p., 2010.

[FOU 16a] FOURNIER, M., C. LARRUE, M. AlEXANDER, D. HEGGER, M. BAKKER, M. PETTERSSON, A. CRABBE, H. MEES, AND A. CHORYNSKI. «Flood risk mitigation in Europe: how far away are we from the aspired forms of adaptive governance? » Ecology and Society 21(4):49, 2016.

[FOU 16b] FOURNIER M., BONNEFOND M., « Regulation of rights and uses in bottom valleys: complex arrangements between environmental public policies and private land uses. The case of "L'île Saint-Aubin" (Angers, France) », 10th Annual Conference of the International Academic Association for Planning, Law and Property Rights, Berne, 2016. 
[GIB 09] GIBOU C., BLANC M., FOUCART J., «Transactions sociales et sciences de l'homme et de la société », Pensée plurielle, 1/2009 (n²0), p. 7-11), 2009.

[GRA 12] GRALEPOIS, M., DALUZEAU, J., OGER, C., «La résilience face à la normativité et la solidarité des territoires », EchoGéo, n²4, 2012.

[HUY 08] HUYGHUES DESPOINTES, F., Des barrages au patrimoine mondial : la Loire comme objet d'action publique, Thèse de doctorat en aménagement de l'espace-urbanisme, Université de Tours, 2008.

[KNO 01] KNOEPFEL P., KISSLING-NAEF I., VARONE F., (eds.), « Institutionelle Regime für natürliche Ressourcen: Boden, Wasser und Wald / Régimes institutionnels de ressources naturelles : analyse comparée du sol, de l'eau et de la forêt », Bâle \& Francfort, Helbing \& Lichtenhahn.

[LAS 07] LASCOUMES P. \& LE GALES P., Sociologie de l'action publique, Ed. Armand Colin, 2007.

[LEL 05] LELOUP F., MOYART L., PECQUEUR B., «La gouvernance territoriale comme nouveau mode de coordination territoriale », Géographie Économie Société, 2005/4 - Vol. 7, 2005.

[LHO 10] LHOMME, S., SERRE D., DIAB Y., LAGANIER R., «Les réseaux techniques face aux inondations ou comment définir des indicateurs de performance de ces réseaux pour évaluer la résilience urbaine. » Bulletin de l'association des géographes français : pp. 487-502., 2010.

[MEL 08] MELE, P.ET LARRUE C. (Coord), Territoires d'action. Aménagement, urbanisme, espace, L'Harmattan, 2008.

[MEL 17] MELOT, R., Droits de propriété et planification foncière. Une perspective de sociologie juridique, Mémoire d'Habilitation à Diriger les Recherches en Sociologie, soutenue le 3 mars 2017 à l'Université de Tours, 2017.

[MOR 12] MORISSEAU, G, « Le quartier périurbain de la Bouillie (Blois) : les nouveaux paysages du risque », Projets de paysage, 2012.

[NAR 01] NAHRATH S., La mise en place du régime institutionnel de l'aménagement du territoire en Suisse entre 1960 et 1990. Thèse de doctorat IDHEAP- Université de Lausanne, 2003.

[NEE 06] NEEDHAM B., Planning, Law and Economics The rules we make for using land, The RTPI Library Series

[NOV 02] NOVEMBER, V., 2002, Les territoires du Risque. Le risque comme objet de réflexion géographique, Peter Lang, 2006.

[NOV 08] NOVEMBER V., Spatiality of risk. Environment and Planning A, n 40 p. 1523-1527, 2008.

[PAS 07] PASQUIER R., SIMOULIN V. ET WEISBEIN J., «La gouvernance territoriale. Pratiques, discours et théories ». Droit et Société vol 44, L.G.D.J. Ed. Paris, 2007.

[PIG 17] PIGEON P., «Risque digue : une justification à la relecture systémique et géopolitique des risques environnementaux », L'Espace Politique [En ligne], 24 | 2014-3, 2017.

[REG 12] REGHEZZA M., RUFAT S., DJAMENT-TRAN G., LE BLANC A. et LHOMME S. «What Resilience Is Not: Uses and Abuses ». Cybergeo : European Journal of Geography [En ligne], 2012.

[REG 06] REGHEZZA M., Réflexions sur la vulnérabilité métropolitaine. La métropole parisienne face au risque de crue centennale. Thèse de doctorat, Université de Paris X-Nanterre, 384 p., 2006

[REM 13] REMY, J., FOUCART, J., « La transaction : une manière de faire de la sociologie. Entretien pour Pensée plurielle », Pensée plurielle 2013/2 (n³3-34), p. 35-51., 2013.

[REM 05] REMY J., « Négociations et transaction sociale », Négociations, n 3, pp. 85-95, 2005.

[REM 91] REMY J., VOYE, L. ET SERVAIS, E. (1991, 1re éd. 1978). Produire ou reproduire? Une sociologie de la vie quotidienne. Bruxelles : De Boeck, 1991.

[ROD 10] RODE S., « De l'aménagement au ménagement des cours d'eau : le bassin de la Loire, miroir de l'évolution des rapports entre aménagement fluvial et environnement », Cybergeo : European Journal of Geography [En ligne], document 506, URL : http://cybergeo.revues.org/23253 ; DOI : 10.4000/cybergeo.23253, 2010.

[ROD 08] RODE S., «La prévention du risque d'inondation, facteur de recompositions urbaine ? L'agglomération de Blois et le déversoir de la Bouillie », L'Information Géographiques 2008/4 (Vol.72), p.6-26, 2008. DOI 10.3917/lig.724.0006.

[SER 11] SERRE D., La ville résiliente aux inondations - Méthodes et outils d'évaluation. Mémoire d'Habilitation à Diriger les Recherches, Université Paris-Est, 173 p., 2011. 
[SER 14] SERVAIN S., «L'aménagement des villes et la gestion des risques d'inondation post 1950. L'exemple de la Loire» in Urbanisation et inondation : outils de réconciliation et de valorisation, coord. Scarwell H-J, Schmitt C., Salvador P-G, presses du Septentrion, Collection «Environnement et société », p.95-124, 2014.

[TOU 12] TOUBIN M., LHOMME S., DIAB Y., SERRE D. ET LAGANIER R., « La Résilience urbaine : un nouveau concept opérationnel vecteur de durabilité urbaine?», Développement durable et territoires [En ligne], Vol. 3, $\mathrm{n}^{\circ}$ 1 | Mai 2012, mis en ligne le 11 juin 2012, 2012.

[TOR 11] TORRE A., «Les processus de gouvernance territoriale. L'apport des proximités », POUR, Juin, N²09-210, 2011.

[VAL 12] VALETTE J-P., " Loire - Blois - Reconquête du bras de décharge du déversoir de « la bouillie », Symposium européen problèmes actuels de la protection contre les inondations, Paris - Orléans 28-29-30 mars 2012.

[VIL 02] VILLENAVE Johanna, Etudes sur l'évolution de l'usage et sur la perception des acteurs locaux de l'île Saint Aubin, (Angers, Maine-et-Loire) en vue d'une gestion intégrée. 2002. 